\title{
Diseño de un instrumento de preferencias vocacionales en Administración, Materiales y Biotecnología \\ Design of an Instrument for Vocational Preferences in Administration, Materials and Biotechnology
}

\author{
Alejandra Alfaro-Barquero y Sonia Chinchilla-Brenes \\ Instituto Tecnológico de Costa Rica, Costa Rica
}

\begin{abstract}
Resumen
Este estudio buscó diseñar y evaluar una prueba de preferencias vocacionales para las carreras de Administración de Empresas, Ingeniería en Materiales e Ingeniería en Biotecnología en el Instituto Tecnológico de Costa Rica (ITCR). Para ello, se construyó previamente el perfil vocacional de cada carrera a partir del cual se redactaron los ítems de la prueba. Participaron 480 estudiantes con una media de edad de 22.07 años, 53.19\% mujeres y 46.81\% hombres. Se obtuvo información del perfil de cada carrera por parte de estudiantes y docentes. Posteriormente, la revisaron grupos de expertos. A partir del perfil definido, se redactaron los ítems de la prueba. Luego, se evaluaron sus características psicométricas con análisis factoriales. Se obtuvieron adecuados indicadores de bondad de ajuste. La prueba vocacional incluyó tres escalas: habilidades, intereses y tareas vocacionales. La escala de habilidades evaluó seis factores: lógico-matemática, física, química, biológica, liderazgo y autorregulación-disciplina. Por su parte, las escalas de tareas e interés se utilizaron para definir las preferencias por los tres factores evaluados: Administración, Materiales y Biotecnología. Finalmente, se evidenciaron diferencias según sexo, carrera y nivel de satisfacción vocacional.
\end{abstract}

Palabras clave: preferencias vocacionales, intereses vocacionales, tareas vocacionales, habilidades, ingeniería

Alejandra Alfaro-Barquero y Sonia Chinchilla-Brenes; Departamento de Orientación y Psicología, Instituto Tecnológico de Costa Rica, Costa Rica.

La correspondencia en relación con este artículo se dirige a Alejandra Alfaro-Barquero, Departamento de Orientación y Psicología, Instituto Tecnológico de Costa Rica, Dirección electrónica: alealfaro@itcr. ac.cr, y a Sonia Chinchilla-Brenes; Departamento de Orientación y Psicología, Instituto Tecnológico de Costa Rica, dirección electrónica: schinchilla@itcr.ac.cr 


\begin{abstract}
:
This study sought to design and evaluate a vocational preference test for Business Administration, Materials Engineering and Biotechnology Engineering majors at the Instituto Tecnológico de Costa Rica (ITCR). For this goal, first of all a vocational profile of each career was constructed, from which the test items were written. 480 students took part, with an average age of 22.07 years, $53.19 \%$ of them women and $46.81 \%$ men. Descriptions of the profile of each career were collected by students and teachers, and later analyzed by expert groups. From the defined profile, items were drawn up and later evaluated with factor analysis, for the selection of the items, obtaining adequate indicators of goodness of fit. The vocational test includes three factors: Skills, Interests and Vocational Tasks. In turn, the Skills scale evaluated six areas: Mathematics, Chemistry, Physics, Biology, Leadership and Self-regulationdiscipline. For its part, the scale of preferences contemplated the three areas evaluated: Administration, Materials and Biotechnology. On the other hand, there were differences according to sex, major and level of vocational satisfaction.
\end{abstract}

Keywords: Vocational Preferences, Vocational Interests, Vocational Tasks, Skills, Engineering

La orientación vocacional es un proceso de educación para la vida profesional. Implica la toma de decisiones autónoma con visualización a futuro del rol social (Cepero, 2009; Müller, 1992) y el análisis de las opciones profesionales (carreras, ocupaciones y demanda laboral) en concordancia con las características personales: intereses, habilidades y preferencias (Bohoslavsky, 1984).

En el proceso de toma de decisiones vocacionales intervienen variedad de factores tales como la orientación y la seguridad vocacional, la condición social, la demanda laboral, las destrezas ocupacionales, el colegio de procedencia y el prestigio social de la carrera; los intereses, las preferencias y el sexo constituyen las más relevantes (Álvarez, Aguilar, Fernández \& Sicilia, 2014; Carrasco, Zúñiga \& Espinoza, 2014; Cepero, 2009; Espíndola, 2015; Hernández, 2001; Herrera \& Burgoa, 2012).

Para este estudio, son especialmente importantes las características del individuo en la elección vocacional. Se parte de un enfoque psicológico de la orientación vocacional (Cepero, 2009). Desde esta perspectiva, Holland (1994) propuso seis tipologías de personalidad, base de la prueba Self-Directed Search (SDS). Dosil y Fernández (2001, en Cepero, 2009) confirmaron su estructura factorial: realista, investigativa, artística, social, emprendedora y convencional

La teoría de Holland plantea que la elección de carrera es una expresión de la personalidad en el quehacer cotidiano. Los individuos, que se desempeñan en un campo profesional específico, tienen estilos de comportamiento, rasgos de personalidad y modos de vida similares (Urribarrí, Flores \& Añez, 2015). La adecuada correspondencia entre las características del individuo y el perfil vocacional de la carrera se reflejará en el grado de satisfacción vocacional (Benítez, 2010).

Si bien se ha encontrado que los estudiantes de ingeniería presentan elementos en común, tales como el interés por la ciencia, la investigación, la tecnología, una perspectiva pragmática, búsqueda de soluciones innovadoras a problemas, habilidad lógico-matemática, creatividad, autodisciplina y habilidades investigativas (Alfaro-Barquero \& Chinchilla-Brenes, 2016, 2017), se plantea que los tipos de personalidad propuestos por Holland (1994) son muy generales y ofrecen pocos criterios para caracterizar las áreas científico-tecnológicas (Alfaro-Barquero \& Chinchilla-Brenes, 2016, 2017). 
Cada disciplina involucra intereses propios y diferentes requerimientos en las habilidades; por ejemplo, Computación implica un mayor grado de habilidad lógico-matemática, no así de habilidad química o física, mientras que Electrónica requiere destrezas en las tres áreas (Alfaro-Barquero \& Chinchilla-Brenes, 2016, 2017).

Por lo tanto, los estudiantes, que se forman en una carrera común, compartirán entre sí afinidades por intereses en temáticas y tareas profesionales, así como ciertas habilidades que les ofrezcan mayores oportunidades de desarrollo personal y laboral. Al mismo tiempo, estos rasgos les permitirán diferenciarse de estudiantes de otras áreas.

En relación con las preferencias vocacionales, varios estudios muestran diferencias según sexo (Echavarri, Godoy \& Olaz, 2007; Fogliatto, Pérez, Olaz \& Parodi, 2003; Martínez \& Ángeles 2016; Martínez-Martínez et al., 2016; Montero, 2005).

Según Rosado y María (2012), los estereotipos de género afectan las expectativas de las mujeres, quienes tienden a inclinarse por ocupaciones con menores oportunidades laborales. Por ejemplo, en Costa Rica, el desempleo femenino alcanza el 15\% y duplica el porcentaje masculino (Adelfang, 2016; Donoso-Vázquez, 2012; Rosado \& María, 2012). Además, las mujeres evidencian menor participación (33\%) en el campo científico-tecnológico (Rodríguez, 2016).

Desde el punto de vista de las preferencias vocacionales, los hombres muestran predilección por las áreas mecánica, tecnológica, electrónica, construcción, actividades realistas, cálculo y ciencias básicas y las mujeres se inclinan por las áreas de comunicación, diseño industrial, jurídica, humanística, asistencial, sanitaria y artística (Alfaro-Barquero \& Chinchilla-Brenes, 2016 y 2017; Fogliatto, Pérez, Olaz \& Parodi, 2003; Martínez \& Ángeles 2016; Martínez-Martínez et al., 2016; Montero, 2005).

Echavarri, Godoy y Olaz (2007) encontraron que los hombres muestran mejor desempeño en razonamiento verbal, abstracto y cálculo y las mujeres, en las pruebas de ortografía y lenguaje. Además, Arcega (2016) señaló que los hombres sobresalen en inteligencia espacial y habilidad lógico-matemática (área ingenieril) y las mujeres, en el cálculo numérico (estadística, contabilidad, etc.).

Por su parte, Alfaro-Barquero y Chinchilla-Brenes $(2016,2017)$ encontraron que los hombres se perciben con mayores habilidades físico-mecánicas y de razonamiento lógico, mientras que las mujeres lo hacen en autorregulación-disciplina y habilidades sociales.

Dichas autoras (Alfaro-Barquero \& Chinchilla-Brenes, 2016, 2017) identificaron cinco habilidades asociadas con la ingeniería: razonamiento lógico matemático, físico-mecánico, visoespacial, liderazgo y autorregulación-disciplina. El razonamiento lógico- matemático refiere a la destreza para comprender, analizar y resolver problemas matemáticos en áreas de aritmética, álgebra, funciones y geometría, mientras que la físico-mecánica implica comprender, analizar y resolver fenómenos físicos asociados con los procesos de movimiento mecánico y transferencia de calor.

La habilidad visoespacial alude a la facilidad para imaginar y ordenar objetos en el entorno, la ubicación espacial, el diseño de modelos y la facilidad para armonizar colores y diseños (Alfaro-Barquero \& Chinchilla-Brenes, 2016 y 2017). 


\section{A. Alfaro-Barquero y S. Chinchilla-Brenes}

Finalmente, la autorregulación-disciplina corresponde a la destreza para realizar tareas con independencia, proactividad, dedicación, persistencia y responsabilidad, mientras que el liderazgo refiere a la habilidad para las relaciones interpersonales, la dirección de proyectos y la capacidad para trabajo en equipo (Alfaro-Barquero \& Chinchilla-Brenes, 2016, 2017).

En relación con los intereses, muchos autores han propuesto clasificaciones e instrumentos vocacionales: la Escala de Intereses Vocacionales de Kuder, (Woyno \& Oñoro, 2005), el Test Explora (Martínez, 2016) y el Cuestionario de Áreas de Intereses Básicos Académico Profesionales (Hernández, 2001). No obstante, incluyen pocos criterios para discriminar específicamente en el área de las ingenierías.

Algunos factores que afectan la elección vocacional son la poca divulgación de carreras no tradicionales o la resistencia del contexto hacia estas (Restrepo, Carvajal \& Roldán 2016), las pruebas de admisión (Carrasco, Zúñiga \& Espinoza, 2014), la condición socioeconómica (Estrada, 2011; Sevilla, Puerta \& Dávila, 2010) y las carencias de orientación vocacional en instituciones educativas (Barrera, 2016).

Ello repercute a nivel universitario, porque se han encontrado incongruencias entre la carrera elegida con los intereses de los estudiantes (Vargas \& Huamán, 2017). Por ejemplo, en el Instituto Tecnológico de Costa Rica (ITCR) se recibieron 1380 solicitudes de cambio de carrera entre el 2014 y 2016 (R. Acuña, comunicación personal, 01 de junio, 2016).

Por lo anterior, este estudio surge como una necesidad de fortalecer los procesos vocacionales que ofrece el ITCR y retoma las investigaciones previas desarrolladas por Alfaro-Barquero y ChinchillaBrenes $(2016,2017)$, en las cuales se definieron los perfiles vocacionales de seis carreras de ingeniería y se construyó una prueba de preferencias vocacionales.

Dichas autoras elaboraron escalas de intereses y tareas para cada carrera (Electrónica, Computación, Mantenimiento Industrial, Diseño Industrial, Construcción y Producción Industrial) y las habilidades de razonamiento lógico, físico-mecánico, viso-espacial, liderazgo y autorregulación-disciplina. La mayoría de las escalas mostraron indicadores psicométricos aceptables y se encontraron diferencias significativas según sexo, nivel de satisfacción vocacional y carrera (Alfaro-Barquero \& Chinchilla-Brenes, 2016, 2017).

La presente investigación se orientó a la construcción de nuevas escalas para el instrumento psicométrico de Tareas, intereses y habilidades en el área de ingeniería en el ITCR (Alfaro-Barquero \& Chinchilla-Brenes, 2016, 2017) para las carreras de Administración de Empresas y las ingenierías en Materiales y Biotecnología.

Con este propósito se utilizó un enfoque de investigación mixto, que combinó estrategias cualitativas, para la elaboración del perfil vocacional, así como estrategias cuantitativas en la construcción y la validación de las escalas del instrumento psicométrico. Asimismo, se realizaron análisis de varianza según carrera, satisfacción vocacional y sexo.

El perfil vocacional se conceptualizó a partir de la percepción de docentes y estudiantes. Contempló cuatro componentes: descripción de la carrera, tareas ocupacionales, intereses y habilidades. La prueba de preferencias vocacionales incluyó las variables tareas, intereses y habilidades para las carreras de la muestra (Alfaro-Barquero \& Chinchilla-Brenes, 2016, 2017). 
Cabe aclarar que el perfil vocacional se dirige a estudiantes en proceso de elección de carrera y considera rasgos de personalidad, intereses, aptitudes o habilidades (Díaz-Barajas, Morales-Rodríguez \& Amador- Zavala, 2009). Se diferencia del perfil profesional, porque este último es la base del diseño curricular acorde con las demandas del mercado laboral y se enfoca en la definición de competencias y la promoción de egresados (Martelo, Jiménez-Pitre \& Quintana, 2018).

\section{Método}

Este estudio implementó una metodología mixta de investigación, que integra procedimientos de recolección y análisis de datos cualitativos y cuantitativos (Pacheco \& Blanco, 2015). Las estrategias cualitativas se utilizaron en la elaboración de los perfiles vocacionales en la primera fase de la investigación.

Por su parte, las estrategias cuantitativas se implementaron para el diseño de las escalas de tareas e intereses de Administración de Empresas y las ingenierías en Materiales y Biotecnología, así como la revisión y la ampliación de la escala de habilidades del instrumento Tareas, intereses y habilidades en el área de Ingeniería en el ITCR (Alfaro-Barquero \& Chinchilla-Brenes, 2016, 2017). Para ello, se incorporaron procedimientos para evaluar las características psicométricas del instrumento y posteriores análisis de varianza.

\section{Participantes}

La población estuvo constituida por estudiantes activos que cursaron asignaturas a partir del sétimo semestre y por docentes del ITCR de Administración de Empresas (AE), las Ingenierías en Materiales (IM) y Biotecnología (IB). La población aproximada total fue de 591 estudiantes en AE, 132 en IB y 184 en IM.

Se contó con tres submuestras, una para cada fase de recolección de datos: 1. fase de construcción del perfil vocacional, 2. Fase de elaboración del instrumento vocacional y pilotaje y 3. Aplicación final del instrumento.

En la fase de construcción del perfil vocacional, según criterio de expertos, se trabajó previamente con dos muestras; la primera se conforma por 100 estudiantes (37 IM, 33 IB y 30 AE) con el objetivo de recabar información sobre las carreras. Se seleccionó el grupo de estudiantes de cada carrera, con mayor número de matrícula, ubicado en el último semestre del plan de estudios. Posteriormente, se seleccionó el grupo de expertos: 12 estudiantes (2 AE, 5 IB y 5 IM), así como 13 docentes ( 3 AE, 4 IB y 6 IM).

Los criterios de inclusión para estudiantes expertos fueron los siguientes: ser estudiante avanzado de la carrera, obtener un promedio ponderado general superior al percentil 75 de la carrera y sentirse satisfecho con su elección vocacional.

Previamente, se solicitó al Departamento de Admisión y Registro la lista de estudiantes activos con sus respectivos promedios de notas. Se preseleccionó un grupo de 10 estudiantes por carrera con los promedios más altos y mayor avance en la carrera. Finalmente, participaron como expertos los estudiantes que indicaron tener altos niveles de satisfacción y que voluntariamente accedieron a colaborar con la investigación. 


\section{A. Alfaro-Barquero y S. Chinchilla-Brenes}

Los criterios de inclusión de los docentes fueron los siguientes: a) tener, al menos, dos años de experiencia docente; $b$ ) haber obtenido en el último año una calificación del desempeño igual o superior a 80 y c) preferible con experiencia laboral en su área de especialidad (al menos, dos años). Los docentes fueron seleccionados por los directores y los coordinadores de cada carrera, según los criterios indicados.

En la segunda fase se elaboró una versión preliminar del instrumento vocacional con ítems redactados a partir del perfil vocacional. Posteriormente, se realizó el pilotaje de la prueba vocacional con un total de 83 estudiantes ( $32 \mathrm{AE}, 26$ IB y 25 IM), que se encontraban cursando asignaturas del quinto semestre del plan de estudios. Los grupos en los que se aplicó el instrumento se eligieron al azar. La edad promedio de la muestra fue $19.85(D E=1.4), 63.9 \%$ eran mujeres, $36.1 \%$, hombres.

En la tercera fase de aplicación completaron el instrumento 268 estudiantes (82 IB, 98 IM y 95 $\mathrm{AE}$ ), que cursaban asignaturas ubicadas a partir del sétimo semestre. Los grupos en los que se aplicó el instrumento se seleccionaron al azar. La edad promedio fue de $22.07(D E=2.89)$. El 52.7\% fue mujeres y el $47.3 \%$, hombres. El 59.5\% procedía de instituciones públicas y el $85.5 \%$, de colegios académicos. La distribución de las muestras anteriormente detallada se resume en la tabla 1.

\section{Tabla 1.}

\section{Distribución de la muestra según fases}

\begin{tabular}{|c|c|c|c|c|c|c|}
\hline \multirow[b]{2}{*}{ Carreras } & \multicolumn{2}{|c|}{$\begin{array}{l}\text { Fase I. } \\
\text { Elaboración del perfil } \\
\text { vocacional }\end{array}$} & $\begin{array}{c}\text { Fase II. } \\
\text { Construcción } \\
\text { prueba vocacional y } \\
\text { Pilotaje }\end{array}$ & $\begin{array}{c}\text { Fase III: } \\
\text { Aplicación final } \\
\text { prueba vocacional }\end{array}$ & \multirow[b]{2}{*}{$\begin{array}{c}\text { Total } \\
\text { estudiantes }\end{array}$} & \multirow[b]{2}{*}{$\begin{array}{c}\text { Total } \\
\text { docentes }\end{array}$} \\
\hline & $\begin{array}{l}\text { A plic a ción } \\
\text { cuestionarios }\end{array}$ & $\begin{array}{c}\text { Estudiantes } \\
\text { expertos }\end{array}$ & Total & Total & & \\
\hline $\mathrm{AE}$ & 30 & 2 & 32 & 95 & 159 & 3 \\
\hline IB & 33 & 5 & 26 & 92 & 156 & 4 \\
\hline IM & 37 & 5 & 25 & 98 & 165 & 6 \\
\hline Total & 100 & 12 & 83 & 285 & 480 & 13 \\
\hline
\end{tabular}

Nota: AE: Administración de Empresas, IB: Ing. en Biotecnología, IM: Ing. en Materiales

\section{Instrumentos}

Se utilizaron cuatro pruebas: a) el instrumento de recolección de información sobre tareas, intereses y habilidades según carrera (Alfaro-Barquero \& Chinchilla-Brenes, 2017); b) las escalas de habilidades y actividades incluidas en la prueba Self-Directed Search de Holland (1994); c) la escala de Evaluación de satisfacción vocacional (Alfaro-Barquero \& Chinchilla-Brenes, 2017) y c. el instrumento de Tareas, intereses y habilidades en el área de ingeniería en el ITCR para las carreras de AE, IB e IM. 
El instrumento de recolección de información sobre tareas, intereses y habilidades fue desarrollado por Alfaro-Barquero y Chinchilla-Brenes (2017). Es un cuestionario abierto de aplicación individual, que solicita información sobre el perfil vocacional de la carrera: definición y áreas profesionales-ocupacionales incorporadas en el plan de estudios, tareas vocacionales, las habilidades e intereses requeridos, así como los contenidos de ciencias básicas de mayor importancia para la disciplina.

Se utilizaron también las escalas de habilidades y actividades de la prueba Self-Directed Search de Holland (1994) con índices de confiabilidad que oscilaron entre .72 y .92 (Hansen, 2004). Cada una de las escalas del SDS incluye seis componentes, que corresponden a las tipologías de personalidad de Holland, con 11 ítems para cada escala y opción de respuesta dicotómica (Sí o No).

Por su parte, el instrumento Evaluación de Satisfacción Vocacional (Alfaro-Barquero \& ChinchillaBrenes, 2017) incluyó ocho ítems que miden el nivel de satisfacción con la profesión elegida y la visualización a futuro de su rol profesional con escala de respuesta de 1 a 5 , de menor a mayor grado de acuerdo. Dicha escala reportó un coeficiente de confiabilidad alfa de Cronbach de .87.

Finalmente, el instrumento de Tareas, intereses y habilidades en el área de ingeniería en el ITCR es un instrumento de evaluación de preferencias vocacionales. Las preferencias vocacionales son la manifestación explícita sobre el grado de predilección por una o varias actividades o profesiones, en concordancia con las características de personalidad y los determinantes contextuales (Cepero, 2009).

El instrumento de Tareas, intereses y habilidades en el área de ingeniería en el ITCR incluyó tres escalas: tareas, habilidades e intereses. Este instrumento abarcó inicialmente 167 ítems y 81 en su versión final. Dichas escalas presentaron coeficientes de confiabilidad alfa de Cronbach que oscilaron entre .78 y .97. Cada ítem tenía opción de respuesta numérica en escala de 1 a 6 , de menor a mayor grado, de acuerdo con cada afirmación.

Las escalas de intereses y tareas contemplaron tres áreas: Ingeniería en Materiales, Ingeniería en Biotecnología y Administración de Empresas. Por su parte, las habilidades incorporaron seis subescalas, cuatro de la escala original de Alfaro-Barquero y Chinchilla-Brenes (2017): lógico matemático, física, liderazgo y autorregulación-disciplina. Además, se incluyeron dos escala: química y biológica.

Conceptualmente, las tareas describen las principales actividades ocupacionales que realiza el profesional en cada una de las áreas laborales de la carrera (Alfaro-Barquero \& Chinchilla-Brenes, 2016, 2017).

Los intereses se consideran incentivos del aprendizaje, aquello que despierta agrado o curiosidad y contiene dimensiones cognitivas, afectivas y conductuales (Rodríguez, 2002). La dimensión cognitiva se asocia con la atención que despierta el objeto de interés; la afectiva, con la sensación de disfrute o predilección y la conductual, con acciones concretas de interacción con el tema (Montero, 2005).

Por su parte, las habilidades se definen como el grado de destreza evidenciado por un individuo frente a la resolución de tareas, las cuales son susceptibles de medición a través del desempeño (Super $\&$ Crites, en Montero, 2005). 


\section{Procedimiento}

El proceso de recolección de los datos se llevó a cabo en tres etapas.

Fase de construcción del perfil vocacional. En esta etapa se elaboró el perfil vocacional. Como paso previo, se aplicó el Instrumento de recolección de información sobre tareas, intereses y habilidades según carrera (Alfaro-Barquero \& Chinchilla-Brenes, 2017). La información recopilada fue sintetizada por las investigadoras para la elaboración preliminar del perfil vocacional de cada carrera y, posteriormente, revisada por el grupo de estudiantes y docentes expertos con la técnica de grupo focales. Se realizaron seis sesiones de trabajo con estudiantes y tres reuniones con docentes con una duración de tres horas cada una.

Fase de elaboración del instrumento Tareas, intereses y habilidades para las carreras seleccionadas y pilotaje de su versión preliminar. Una vez concluidos los perfiles vocacionales, se procedió a la selección de los contenidos y la redacción de los ítems para el instrumento vocacional. La prueba vocacional, elaborada para las carreras IM, IB y AE, se aplicó en conjunto con las escalas de habilidades y actividades del SDS de Holland (1994).

La información de los instrumentos fue digitalizada en una base de datos para el análisis de sus características psicométricas con análisis factoriales exploratorios. Como resultado, se eliminaron los ítems que no mostraron adecuados indicadores y se incorporaron mejoras de redacción.

Fase de aplicación final del instrumento. Se aplicó la versión mejorada del instrumento Tareas, intereses y habilidades en el área de ingeniería en el ITCR para las carreras IM, IB y AE, de manera conjunta con la escala Evaluación de satisfacción vocacional, a una muestra de 285 estudiantes.

\section{Análisis de datos}

En la primera fase de investigación, los datos recopilados mediante la aplicación del instrumento de recolección de información sobre tareas, intereses y habilidades, según carrera (Alfaro-Barquero \& Chinchilla-Brenes, 2017), fue digitada e integrada en un único documento con formato Word. Se consideraron las siguientes categorías de análisis: definición de la carrera, áreas profesionales, tareas ocupacionales por área, áreas profesionales afines, intereses y habilidades.

Posteriormente, se convocó a reuniones según carrera al grupo de estudiantes expertos para la definición del perfil vocacional, a partir de las categorías de análisis antes mencionadas. A cada grupo se le presentó por escrito la información obtenida en la fase anterior y se le proyectó en una pizarra con apoyo de equipo de cómputo.

Seguidamente, se leyó el documento. Cada participante brindó su punto de vista sobre el tema y se generaron discusiones sobre los diversos argumentos para que los participantes llegaran a estar de acuerdo sobre la redacción del contenido del texto en cada una de las categorías de análisis. Se consideraron pertinencia, estructura, contenido y aspectos de redacción. Durante el proceso, las investigadoras moderaron la discusión, introdujeron los cambios al documento escrito y promovieron el uso de un léxico accesible a población no experta. 
El perfil vocacional, resultado del proceso anterior, fue revisado y mejorado en sesiones de discusión grupal con los docentes expertos de cada carrera. Se siguió la misma metodología utilizada con el grupo de estudiantes.

Por su parte, la versión piloto del instrumento Tareas, intereses y habilidades en el área de ingeniería en el ITCR para las carreras IM, IB y AE, las escalas de habilidades y actividades del SDS de Holland (1994) y la escala de Evaluación de satisfacción vocacional (Alfaro-Barquero \& Chinchilla-Brenes, 2017), se aplicaron a la muestra de 83 estudiantes. Los resultados se evaluaron con procedimientos psicométricos mediante análisis de factores exploratorios y el cálculo del coeficiente de confiabilidad alfa de Cronbach. Todo lo anterior se propuso como objetivo el implementar mejoras al instrumento previo a la aplicación final.

En la fase final, se aplicaron los siguientes instrumentos a una muestra de 285 estudiantes: 1.Tareas, intereses y habilidades en el área de ingeniería en el ITCR para las carreras IM, IB y AE; y 2. Evaluación de satisfacción vocacional (Alfaro-Barquero \& Chinchilla-Brenes, 2017).

Para el análisis de los datos se implementaron procedimientos del Análisis Factorial Confirmatorio con el propósito de evaluar con mayor rigurosidad cuán convenientes son los indicadores utilizados en la medición de factores latentes (Cea, 2002). Para ello, se utilizó el paquete estadístico EQS 6.1 con el método de estimación de Máxima Verosimilitud, porque varios autores "sugieren que la estimación de máxima verosimilitud es razonablemente robusta para muchas de las violaciones del supuesto de normalidad" (Cea, 2002, p.521). Lo anterior permitió seleccionar los ítems más apropiados y confirmar la estructura factorial.

Se propuso confirmar la presencia de dos factores: las preferencias vocacionales y las habilidades. Las preferencias vocacionales se definieron a partir de las puntuaciones de las tareas e intereses en tres áreas: Ingeniería en Materiales, Administración de Empresas e Ingeniería en Biotecnología. Para las habilidades se planteó la existencia de seis factores: matemática, física, biológica, químicas, liderazgo y autorregulación-disciplina.

En la evaluación de los modelos se incluyeron los indicadores de bondad de ajuste CFI (índice de ajuste comparativo), RMSEA (la raíz cuadrada del error de estimación) y la chi cuadrada $\left(\chi^{2}\right)$. Para lograr un buen ajuste, el CFI debe ser igual o superior a .9 y el RMSEA inferior o equivalente a .05, aunque un valor inferior a .08 se considera un porcentaje de error aceptable (Cea, 2002).

Por su parte, la chi cuadrada debe ser pequeña y su probabilidad superior a .05 (Cea, 2002). No obstante, es posible obtener valores inferiores a este criterio si algunas variables incumplen el supuesto de distribución normal (Cea, 2002). Finalmente, se realizó el análisis de varianza (paquete estadístico SPSS) para evaluar diferencias según sexo, satisfacción vocacional y carrera.

\section{Resultados}

Se elaboró el perfil vocacional que se resume en la tabla 2. Se describen las principales características de las áreas de Administración de Empresas, Materiales y Biotecnología. Se consideran definición, tareas ocupacionales, intereses y habilidades. 


\section{A. Alfaro-Barquero y S. Chinchilla-Brenes}

\section{Tabla 2}

\section{Sintesis de perfiles vocacionales}

\begin{tabular}{|c|c|c|c|c|}
\hline Descripción AE & Áreas & Tareas ocupacionales & Áreas afines & Intereses y habilidades \\
\hline $\begin{array}{l}\text { Es una carrera } \\
\text { orientada a formar } \\
\text { profesionales } \\
\text { con capacidad de } \\
\text { participar o dirigir el } \\
\text { proceso administrativo } \\
\text { (planeación, } \\
\text { organización, } \\
\text { dirección y control } \\
\text { de los procesos, } \\
\text { proyectos y recursos) } \\
\text { de una empresa u } \\
\text { organización para } \\
\text { el cumplimiento } \\
\text { eficaz y eficiente de } \\
\text { sus objetivos y la } \\
\text { maximización de las } \\
\text { utilidades dentro del } \\
\text { marco de la ética, la } \\
\text { responsabilidad social } \\
\text { y ambiental. }\end{array}$ & Mercadeo & $\begin{array}{l}\text {-Análisis de estados } \\
\text { financieros de la } \\
\text { empresa (utilidades y } \\
\text { pérdidas). } \\
\text {-Evaluación de } \\
\text { riesgos de inversiones } \\
\text { y rentabilidad de } \\
\text { proyectos. } \\
\text {-Proyecciones de ventas, } \\
\text { presupuesto y efectivo } \\
\text { disponible. } \\
\text {-Estudios de mercado: } \\
\text { población meta, } \\
\text { pronósticos de demanda } \\
\text { y oferta. } \\
\text {-Estrategia de mercadeo } \\
\text { y promoción. } \\
\text {-Evaluación de } \\
\text { satisfacción del cliente. }\end{array}$ & $\begin{array}{l}\text {-Publicidad } \\
\text {-Diseño Industrial y } \\
\text { Gráfico }\end{array}$ & $\begin{array}{l}\text { Intereses: } \\
\text {-Economía y finanzas. } \\
\text {-Desarrollo de personal } \\
\text {-Comportamiento y } \\
\text { relaciones interpersonales } \\
\text {-Comportamiento } \\
\text { empresarial y publicidad } \\
\text { Habilidades: } \\
\text {-Relaciones interpersonales } \\
\text {-Trabajo en equipo } \\
\text {-Expresión oral y escrita } \\
\text {-Proactividad y creatividad } \\
\text {-Liderazgo } \\
\text {-Pensamiento estratégico } \\
\text {-Persuasión } \\
\text {-Empatía } \\
\text {-Matemática }\end{array}$ \\
\hline
\end{tabular}


DISEÑO DE UN INSTRUMENTO DE PREFERENCIAS VOCACIONALES

Tabla 2

Sintesis de perfiles vocacionales

\begin{tabular}{|c|c|c|c|c|}
\hline Descripción AE & Áreas & Tareas ocupacionales & \multirow{2}{*}{$\begin{array}{l}\text { Áreas afines } \\
\text {-Psicología }\end{array}$} & Intereses y habilidades \\
\hline & Gestión de & -Gestión del talento & & \\
\hline & talento humano & humano, clima & -Ingeniería en & \\
\hline & & organizacional y diseño & Seguridad Laboral & \\
\hline & & $\begin{array}{l}\text {-Reclutamiento y } \\
\text { selección de personal. }\end{array}$ & & \\
\hline & & $\begin{array}{l}\text {-Capacitación y } \\
\text { evaluación del personal. }\end{array}$ & & \\
\hline & Contabilidad & -Manejo de libros & -Contaduría & \\
\hline & & contables y legales & -Ingeniería en & \\
\hline & & (teneduría). & Producción Industrial & \\
\hline & & -Elaboración de estados & & \\
\hline & & financieros. & & \\
\hline & & -Registro de & & \\
\hline & & información contable. & & \\
\hline
\end{tabular}

Continúa ... 


\section{A. Alfaro-Barquero y S. Chinchilla-Brenes}

\section{Tabla 2}

\section{Sintesis de perfiles vocacionales}

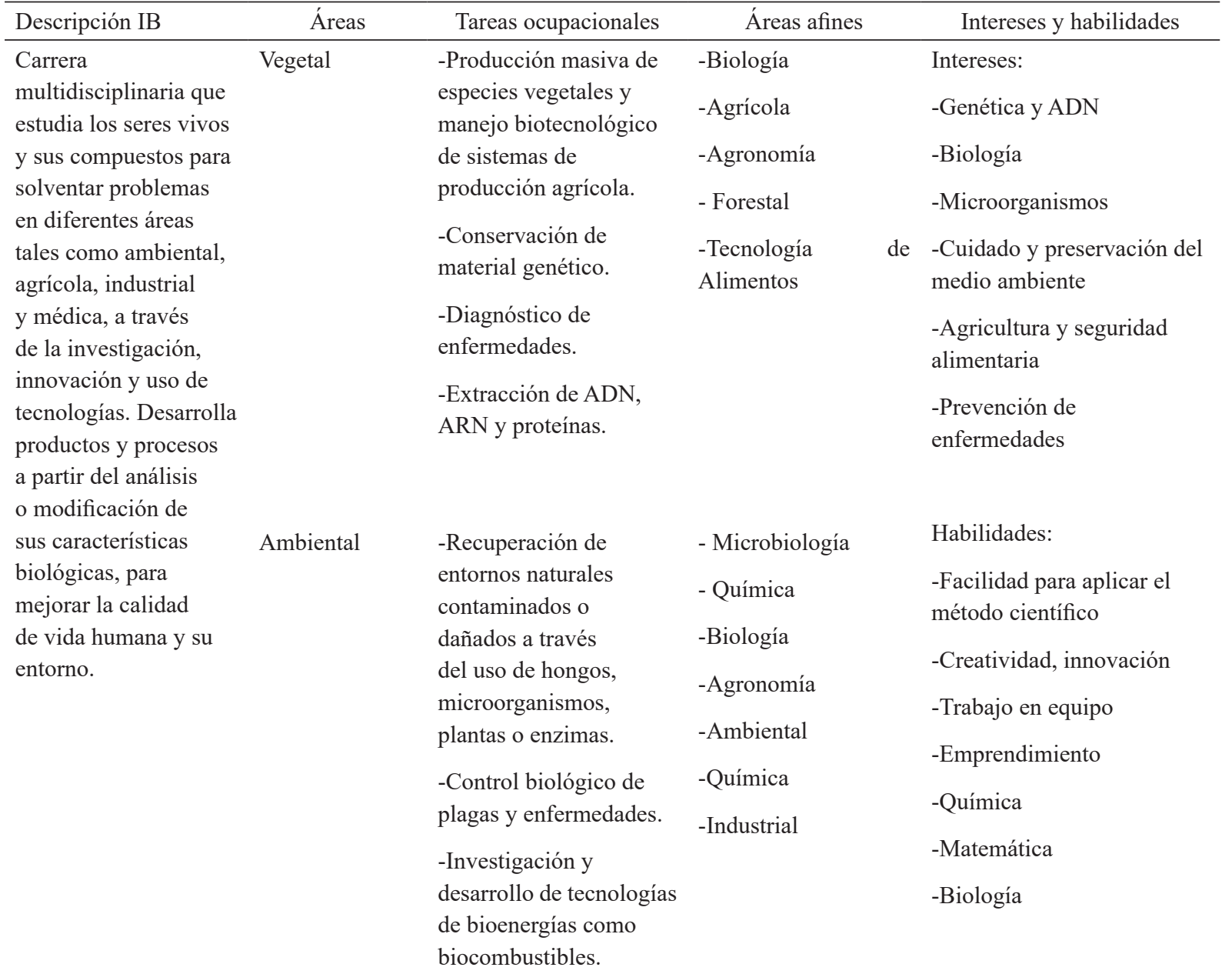


DISEÑO DE UN INSTRUMENTO DE PREFERENCIAS VOCACIONALES

Tabla 2

Sintesis de perfiles vocacionales

\begin{tabular}{|c|c|c|c|c|}
\hline Descripción IB & Áreas & Tareas ocupacionales & Áreas afines & Intereses y habilidades \\
\hline & \multirow[t]{5}{*}{ Biomédica } & -Investigación para & -Farmacia & \\
\hline & & $\begin{array}{l}\text { el desarrollo de } \\
\text { compuestos biológicos }\end{array}$ & -Medicina & \\
\hline & & (antibióticos, enzimas y & -Biología & \\
\hline & & otros). & -Microbiología & \\
\hline & & $\begin{array}{l}\text {-Estudio de } \\
\text { microrganismos ligados } \\
\text { a enfermedades o } \\
\text { aplicaciones de interés. }\end{array}$ & -Veterinaria & \\
\hline
\end{tabular}

Continúa... 


\section{A. Alfaro-Barquero y S. Chinchilla-Brenes}

\section{Tabla 2}

\section{Sintesis de perfiles vocacionales}

\begin{tabular}{|c|c|c|c|c|}
\hline Descripción IM & Áreas & Tareas ocupacionales & Áreas afines & Intereses y habilidades \\
\hline $\begin{array}{l}\text { Carrera orientada } \\
\text { a investigación, } \\
\text { estudio, clasificación, } \\
\text { selección, } \\
\text { conservación y } \\
\text { transformación } \\
\text { de los materiales } \\
\text { tales como metales, } \\
\text { plásticos, cerámicos y } \\
\text { compuestos, a partir } \\
\text { del análisis de su } \\
\text { estructura atómica, } \\
\text { propiedades físicas, } \\
\text { mecánicas y químicas, } \\
\text { así como sus usos, } \\
\text { con el objetivo de } \\
\text { desarrollar productos } \\
\text { utilizados en diversos } \\
\text { ámbitos de la } \\
\text { sociedad. }\end{array}$ & $\begin{array}{l}\text { Procesos } \\
\text { industriales }\end{array}$ & $\begin{array}{l}\text {-Proceso de } \\
\text { transformación de un } \\
\text { material. } \\
\text {-Diseñar, seleccionar y } \\
\text { planificar procesos de } \\
\text { manufactura según tipos } \\
\text { de materiales. } \\
\text {-Detección y control } \\
\text { de fallas y mejora } \\
\text { de procesos de } \\
\text { manufactura. } \\
\text {-Estimación de costos y } \\
\text { selección de materiales } \\
\text { y procesos. } \\
\text {-Fabricación y ensamble } \\
\text { de estructuras metálicas. }\end{array}$ & $\begin{array}{l}\text {-Producción } \\
\text { Industrial } \\
\text {-Industrial. } \\
\text {-Mantenimiento } \\
\text { Industrial. } \\
\text {-Mecánica. } \\
\text {-Química. }\end{array}$ & $\begin{array}{l}\text { Intereses: } \\
\text {-Conocer y mejorar procesos } \\
\text { industriales } \\
\text {-Procesos de fabricación } \\
\text { de materiales (metales, } \\
\text { polímeros, cerámicas y } \\
\text { compuestos) y corrección } \\
\text { de fallas. } \\
\text {-Resistencia de los } \\
\text { materiales al ambiente } \\
\text {-Actualización científica y } \\
\text { tecnológica } \\
\text { Habilidades: } \\
\text {-Investigación } \\
\text {-Trabajo interdisciplinario } \\
\text {-Dibujo técnico } \\
\text {-Aplicar método científico } \\
\text {-Creatividad e innovación } \\
\text {-Análisis y toma de } \\
\text { decisiones } \\
\text {-Física, Matemática, } \\
\text { Química }\end{array}$ \\
\hline
\end{tabular}


Tabla 2

Sintesis de perfiles vocacionales

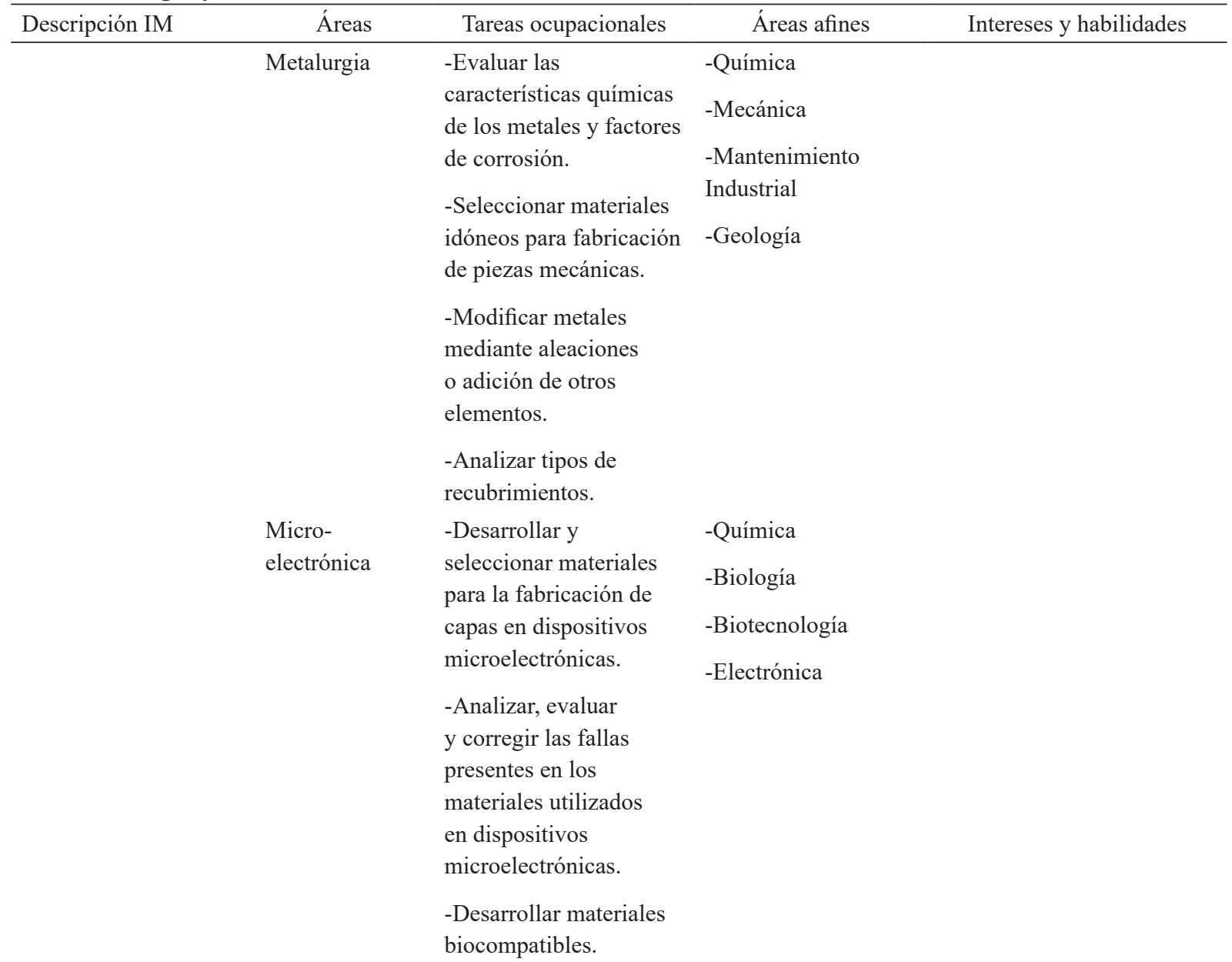

Nota: AE: Administración de Empresas, IM: Ingeniería en Materiales e IB: Ingeniería en Biotecnología.

Además, las cualidades psicométricas de la primera versión del instrumento aplicado en la fase piloto se reflejan en la tabla 3, en la que los resultados del análisis factorial exploratorio de las escalas del SDS evidenciaron porcentajes de varianza total de $48 \%$ para actividades y $45 \%$ para habilidades y coeficientes de confiabilidad que oscilaron entre .57 y .94; valores inferiores a los obtenidos en el instrumento Tareas, intereses y habilidades para las carreras IM, IB y AE (63.27\% para habilidades, $70.26 \%$ para intereses y $76.36 \%$ para tareas) y coeficientes de confiabilidad que variaron entre .69 y .98 . La escala de Evaluación de satisfacción vocacional mostró una varianza explicada de $37.44 \%$ y una confiabilidad de .72 . 
Tabla 3

Resultados de los análisis de factores exploratorios. Fase piloto

\begin{tabular}{|c|c|c|c|c|c|c|c|c|}
\hline Escala & $\begin{array}{l}\text { KMO } \\
>.5 \\
>.75 \\
\end{array}$ & $\begin{array}{l}\text { Varianza } \\
\text { explicada } \\
\text { total }\end{array}$ & $\begin{array}{c}\text { Bartlett } \\
<.05\end{array}$ & $\begin{array}{l}\mathrm{F} \text { a } \mathrm{c} \mathrm{t} \text { o } \mathrm{r} \text { e } \mathrm{s} \\
\text { identificados }\end{array}$ & $\begin{array}{l}\text { Ít e m s } \\
\text { s e gún } \\
\text { escala }\end{array}$ & $\begin{array}{l}\text { Alfa de } \\
\text { Cronbach } \\
\geq .8\end{array}$ & $\begin{array}{l}\text { Varianza } \\
\text { explicada }\end{array}$ & $\begin{array}{c}\text { Ítems } \\
\text { coincidentes } \\
\text { con escalas } \\
\text { teóricas }\end{array}$ \\
\hline \multirow{5}{*}{$\begin{array}{l}\text { Escala de } \\
\text { actividades } \\
\text { de Holland }\end{array}$} & \multirow{5}{*}{.75} & \multirow{6}{*}{$48.1 \%$} & \multirow{6}{*}{$p<.001$} & $\begin{array}{l}\text { Convencional } \\
\text { emprendedor }\end{array}$ & 22 & .94 & $21.6 \%$ & 17 \\
\hline & & & & Realista & 11 & .87 & $10.7 \%$ & 10 \\
\hline & & & & Investigativa & 11 & .83 & $5.9 \%$ & 6 \\
\hline & & & & Artística & 11 & .73 & $4.9 \%$ & 6 \\
\hline & & & & Social & 11 & .57 & $4.9 \%$ & 3 \\
\hline \multirow{5}{*}{$\begin{array}{l}\text { Escala de } \\
\text { habilidades } \\
\text { de Holland }\end{array}$} & \multirow{5}{*}{.67} & & & Investigativa & 11 & .87 & $9.95 \%$ & 10 \\
\hline & & \multirow{4}{*}{$45 \%$} & \multirow{4}{*}{$p<.001$} & Convencional & 11 & .85 & $9.62 \%$ & 7 \\
\hline & & & & Realista & 11 & .86 & $9.54 \%$ & 8 \\
\hline & & & & Emprendedora & 11 & .85 & $9.1 \%$ & 11 \\
\hline & & & & Social & 11 & .83 & $7.6 \%$ & 7 \\
\hline \multirow{5}{*}{$\begin{array}{l}\text { Escala de } \\
\text { habilidades }\end{array}$} & \multirow{5}{*}{.722} & \multirow{5}{*}{$63.27 \%$} & \multirow{5}{*}{$p<.001$} & Físico-química & 15 & .96 & $20.4 \%$ & 14 \\
\hline & & & & Biología & 8 & .97 & $16 \%$ & 7 \\
\hline & & & & Liderazgo & 12 & .92 & $14.9 \%$ & 14 \\
\hline & & & & $\begin{array}{l}\text { Razonamiento } \\
\text { lógico- } \\
\text { matemático }\end{array}$ & 9 & .89 & $7.8 \%$ & 6 \\
\hline & & & & $\begin{array}{l}\text { Autorregulación } \\
\text { y disciplina }\end{array}$ & 8 & .69 & $4 \%$ & 5 \\
\hline
\end{tabular}


Tabla 3

Resultados de los análisis de factores exploratorios. Fase piloto

\begin{tabular}{|c|c|c|c|c|c|c|c|c|}
\hline Escala & $\begin{array}{c}\text { KMO } \\
>.5 \\
>.75\end{array}$ & $\begin{array}{c}\text { Varianza } \\
\text { explicada } \\
\text { total }\end{array}$ & $\begin{array}{l}\text { Bartlett } \\
<.05\end{array}$ & $\begin{array}{c}\text { Factores } \\
\text { identificados }\end{array}$ & $\begin{array}{l}\text { Ítems } \\
\text { según } \\
\text { escala }\end{array}$ & $\begin{array}{l}\text { Alfa de } \\
\text { Cronbach } \\
\geq .8\end{array}$ & $\begin{array}{l}\text { Varianza } \\
\text { explicada }\end{array}$ & $\begin{array}{c}\text { Ítems } \\
\text { coincidentes } \\
\text { con escalas } \\
\text { teóricas }\end{array}$ \\
\hline \multirow{3}{*}{$\begin{array}{l}\text { Escala } \\
\text { intereses }\end{array}$} & \multirow{3}{*}{.857} & \multirow{3}{*}{$70.26 \%$} & \multirow{3}{*}{$p<.001$} & Biotecnología & 14 & .96 & $26.4 \%$ & 14 \\
\hline & & & & Materiales & 13 & .96 & $25.1 \%$ & 12 \\
\hline & & & & $\begin{array}{l}\text { Administración de } \\
\text { Empresas }\end{array}$ & 9 & .95 & $18.7 \%$ & 9 \\
\hline \multirow{3}{*}{$\begin{array}{l}\text { Escala de } \\
\text { tareas }\end{array}$} & \multirow{3}{*}{.831} & \multirow{3}{*}{$76.36 \%$} & \multirow{3}{*}{$p<.001$} & Biotecnología & 20 & .96 & $27.2 \%$ & 20 \\
\hline & & & & $\begin{array}{l}\text { Administración de } \\
\text { Empresas }\end{array}$ & 23 & .98 & $25.5 \%$ & 23 \\
\hline & & & & Materiales & 21 & .98 & $23.6 \%$ & 20 \\
\hline \multirow{2}{*}{$\begin{array}{l}\text { Satisfacción } \\
\text { vocacional }\end{array}$} & \multirow{2}{*}{.73} & \multirow{2}{*}{$37.40 \%$} & \multirow{2}{*}{$p<.001$} & Factor 1 & 6 & \multirow{2}{*}{.72} & $25.26 \%$ & \multirow{2}{*}{8} \\
\hline & & & & Factor 2 & 2 & & $12.18 \%$ & \\
\hline
\end{tabular}

Por su parte, los resultados del análisis factorial confirmatorio de la fase final de aplicación del instrumento Tareas, intereses y habilidades se presentan en la tabla 4, en la cual se indica el número total de ítems de cada escala y los coeficientes de confiabilidad, que oscilaron entre .74 y .95. También, se reporta el valor mínimo de discriminación por ítem (según teoría clásica de los test), que en todos los casos fue superior a 4 . 
Tabla 4

Resultados de la evaluación de los modelos de medición con el análisis factorial confirmatorio $y$ de otras características psicométricas de las subescalas.

\begin{tabular}{|c|c|c|c|c|c|c|c|c|}
\hline Escala & Ítems & $\begin{array}{c}\text { Alfa de } \\
\text { Cronbach }\end{array}$ & $\begin{array}{l}\text { Discriminación } \\
\text { mínima por ítem }\end{array}$ & CFI & RMSEA & $\begin{array}{c}\text { Chi } \\
\text { cuadrado }\end{array}$ & $\begin{array}{l}\text { Coeficiente } \\
\text { factorial } \\
\text { mínima }\end{array}$ & $\begin{array}{c}\text { Coeficiente } \\
\text { factorial } \\
\text { máxima }\end{array}$ \\
\hline $\begin{array}{l}\text { Satisfacción } \\
\text { vocacional }\end{array}$ & 8 & .83 & .43 & .96 & .08 & $\begin{array}{c}48.16 \\
p<.001\end{array}$ & 0.47 & 0.87 \\
\hline $\begin{array}{l}\text { Intereses } \\
\text { Biotecnología }\end{array}$ & 8 & .94 & .61 & .99 & .06 & $\begin{array}{c}39.3 \\
p<.001\end{array}$ & 0.62 & 0.94 \\
\hline $\begin{array}{l}\text { Intereses } \\
\text { Materiales }\end{array}$ & 5 & .96 & .87 & 1 & .03 & $\begin{array}{c}4.99 \\
p=.29\end{array}$ & 0.87 & 0.96 \\
\hline $\begin{array}{l}\text { Intereses } \\
\text { Administración }\end{array}$ & 7 & .94 & .77 & .99 & .05 & $\begin{array}{c}21.8 \\
p=.06\end{array}$ & 0.79 & 0.88 \\
\hline $\begin{array}{l}\text { Habilidad lógico- } \\
\text { matemática }\end{array}$ & 5 & .89 & .64 & .99 & .08 & $\begin{array}{c}8.37 \\
p=.04\end{array}$ & 0.70 & 0.86 \\
\hline Habilidad física & 5 & .94 & .81 & 1 & .00 & $\begin{array}{c}1.13 \\
p=.77\end{array}$ & 0.84 & 0.96 \\
\hline Habilidad química & 5 & .95 & .75 & 1 & .06 & $\begin{array}{c}5.81 \\
p=.12\end{array}$ & 0.78 & 0.97 \\
\hline $\begin{array}{l}\text { Habilidad } \\
\text { biológica }\end{array}$ & 7 & .97 & .77 & 1 & .08 & $\begin{array}{c}36.4 \\
p<.001\end{array}$ & 0.78 & 0.95 \\
\hline $\begin{array}{l}\text { Habilidad } \\
\text { liderazgo }\end{array}$ & 8 & .86 & .57 & .96 & .07 & $\begin{array}{c}51 \\
p<.001\end{array}$ & 0.61 & 0.7 \\
\hline $\begin{array}{l}\text { Habilidad } \\
\text { Disciplina }\end{array}$ & 4 & .78 & .44 & 1 & .02 & $\begin{array}{c}2.15 \\
p=.34\end{array}$ & 0.49 & 0.82 \\
\hline $\begin{array}{l}\text { Tareas } \\
\text { Biotecnología }\end{array}$ & 8 & .96 & .71 & .99 & .08 & $\begin{array}{c}56.7 \\
p<.001\end{array}$ & 0.72 & 0.93 \\
\hline Tareas Materiales & 8 & .97 & .84 & .99 & .07 & $\begin{array}{c}50.5 \\
p<.001\end{array}$ & 0.85 & 0.96 \\
\hline $\begin{array}{l}\text { Tareas } \\
\text { Administración }\end{array}$ & 11 & .97 & .73 & .98 & .06 & $\begin{array}{c}93.69 \\
p<.001\end{array}$ & 0.74 & 0.91 \\
\hline
\end{tabular}

Se obtuvieron indicadores de ajuste favorables: los valores de CFI fueron superiores a .96, los del RMSEA iguales o inferiores a .08 y los del chi cuadrado fueron relativamente pequeños. Asimismo, los coeficientes factoriales que determinan el grado en que el comportamiento de cada ítem es explicado por la variable latente (Cea, 2002), mostraron valores entre .47 y .96, lo que se considera aceptable. 
Una vez realizados los modelos de medición de cada subfactor, se procedió a realizar los análisis confirmatorios para comprobar la estructura factorial de los dos modelos evaluados: a. modelo de preferencias vocacionales y $\mathrm{b}$. modelo de habilidades.

El modelo de preferencias vocacionales se construyó al utilizar como indicadores los puntajes globales de las escalas de tareas e intereses según carrera. En la figura 1 se describen los resultados del análisis confirmatorio para la escala de preferencias vocacionales. Se evidenciaron tres factores: Preferencias por la Ingeniería en Materiales, la Administración de Empresas y la Ingeniería en Biotecnología, todos los cuales correlacionaron entre sí en dirección negativa.

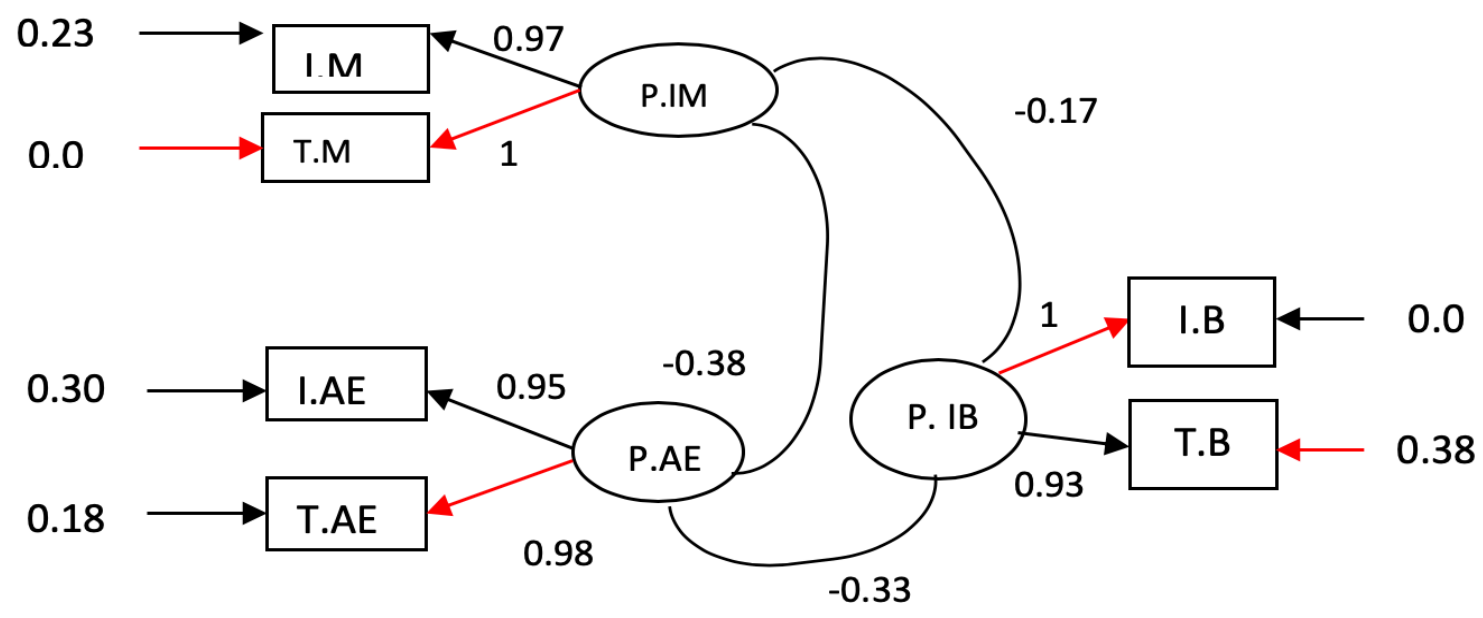

Figura 1. Modelo Factorial Confirmatorio para las preferencias vocacionales. Indicadores de bondad de ajuste: CFI: .99; RMSEA: .08; Chi cuadrada: $\chi^{2}(8)=17.78, p=.01$. P.IM: Preferencias vocacionales Ing. en Materiales; I.M: Intereses Ing. en Materiales; T.M: Tareas profesionales Ing. en Materiales; P.AE: Preferencias vocacionales Adm.de Empresas; I.AE: Intereses Adm.de Empresas; T.AE Tareas profesionales Adm.de Empresas;P.IB: Preferencias vocacionales Ing. en Biotecnología; I.B: Intereses Ing. en Biotecnología; T.B: Tareas profesionales Ing. en Biotecnología.

Por su parte, el modelo de habilidades se elaboró al utilizar parcelas por cada habilidad. Mediante dicho procedimiento los ítems componen una escala y se dividen para obtener dos subpuntuaciones. Cada una de ellas se emplea como indicador para medir la variable latente. Ello permitió obtener un modelo más parsimonioso.

En la figura 2 se detalla el modelo confirmatorio para la escala de habilidades. Se evidenciaron seis variables: liderazgo y disciplina como habilidades de corte más socioemocional, las cuales mostraron una covarianza positiva de .62. Las otras cuatro habilidades de ciencias básicas se asociaron entre sí. La habilidad física mostró alta correlación con matemática y química; a su vez, la habilidad química se correlacionó con las áreas matemática y biológica. 


\section{A. Alfaro-Barquero y S. Chinchilla-Brenes}

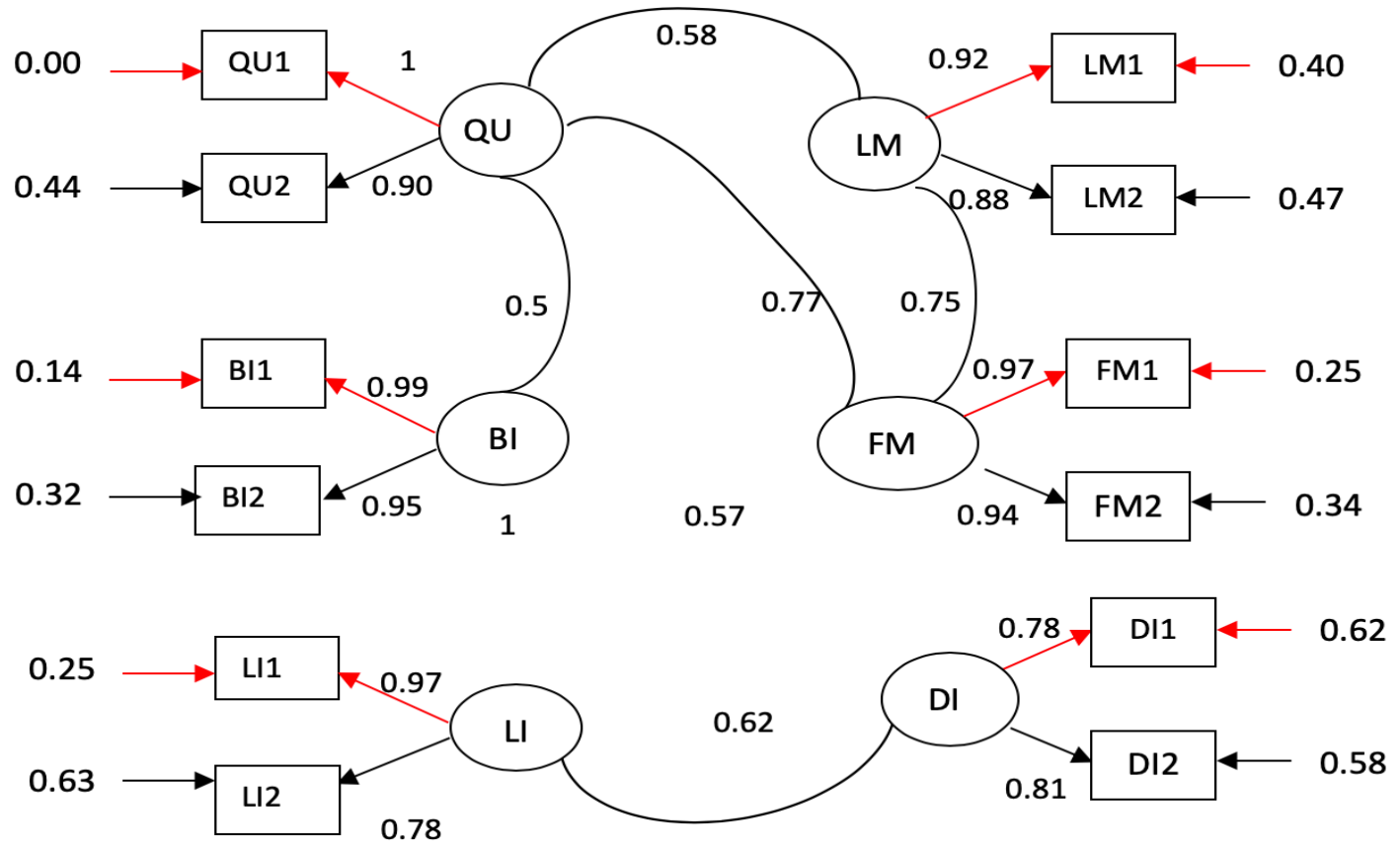

Figura 2. Modelo Factorial Confirmatorio para la escala de habilidades. Indicadores de bondad de ajuste: CFI: .97; RMSEA: 0.08; chi cuadrada: $\chi^{2}(49)=137.59, p<.001$. QU: Química; BI: Biologíca; LM: Lógico-matemático; FM: Físico-mecánica; LI: Liderazgo; DI: Disciplina.

Además, los análisis de varianza, según carrera, evidenciaron diferencias significativas en todas las variables, tal como se muestra en la tabla 5: lógico matemático $\left(\mathrm{F}(2)=48.854, p<.001, \eta_{\mathrm{p}}{ }^{2}=.23\right)$, habilidad física $\left(\mathrm{F}(2)=223.090, p<.001, \eta_{\mathrm{p}}{ }^{2}=.61\right)$, habilidad química $\left(\mathrm{F}(2)=242.935, p<.001, \eta_{\mathrm{p}}{ }^{2}\right.$ $=.64)$, habilidad biológica $\left(\mathrm{F}(2)=391.092, p<.001, \eta_{\mathrm{p}}{ }^{2}=.75\right)$, liderazgo $\left(\mathrm{F}(2)=17.407, p<.001, \eta_{\mathrm{p}}{ }^{2}\right.$ $=.13)$, disciplina $\left(\mathrm{F}(2)=3.646, p<.001, \eta_{\mathrm{p}}{ }^{2}=.03\right)$, intereses $\operatorname{IM}\left(\mathrm{F}(2)=556.595, p<.001, \eta_{\mathrm{p}}{ }^{2}=.81\right)$, intereses IB $\left(\mathrm{F}(2)=237.333, p<.001, \eta_{\mathrm{p}}{ }^{2}=.66\right)$, intereses $\mathrm{AE}\left(\mathrm{F}(2)=180.283, \mathrm{p}<.001, \eta_{\mathrm{p}}{ }^{2}=.53\right)$, tareas IB $\left(\mathrm{F}(2)=242.791, p<.001, \eta_{\mathrm{p}}{ }^{2}=.68\right)$, tareas IM $\left(\mathrm{F}(2)=682.367, p<.001, \eta_{\mathrm{p}}{ }^{2}=.85\right)$, tareas AE (F(2) $\left.=269.27, p<.001, \eta_{\mathrm{p}}^{2}=.64\right)$ y promedio ponderado $\left(\mathrm{F}(2)=30.681, p<.001, \eta_{\mathrm{p}}{ }^{2}=.2\right)$. 
Tabla 5

Análisis de varianza según carrera

\begin{tabular}{lcccccc}
\hline & $\begin{array}{c}\text { Suma de } \\
\text { cuadrados tipo }\end{array}$ & \multicolumn{3}{c}{ Media } & & $\begin{array}{c}\text { Eta al } \\
\text { cuadrado } \\
\text { parcial: } \eta_{p}{ }^{2}\end{array}$ \\
\hline Láriable dependiente & III & $g l$ & cuadrática & $F$ & $p$ & .23 \\
Física & 91.614 & 2 & 45.81 & 34.65 & $<.01$ & .61 \\
Química & 368.335 & 2 & 184.17 & 182.80 & $<.01$ & .64 \\
Biológica & 451.999 & 2 & 226.00 & 206.96 & $<.01$ & .75 \\
Liderazgo & 640.365 & 2 & 320.18 & 350.00 & $<.01$ & .13 \\
Disciplina & 27.792 & 2 & 13.90 & 17.08 & $<.01$ & .13 \\
Intereses Materiales & 5.335 & 2 & 2.67 & 3.64 & .03 & .03 \\
Intereses Biotecnología & 698.760 & 2 & 349.38 & 512.50 & $<.01$ & .81 \\
Intereses Administración & 489.349 & 2 & 244.67 & 231.58 & $<.01$ & .66 \\
Intereses Biotecnología & 316.866 & 2 & 158.43 & 136,16 & $<.01$ & .53 \\
Tareas Materiales & 537.197 & 2 & 268.60 & 254.17 & $<.01$ & .68 \\
Tareas Administración & 734.842 & 2 & 367.42 & 646.57 & $<.01$ & .85 \\
Tareas Promedio ponderado & 388.789 & 2 & 194.39 & 210.57 & $<.01$ & .64 \\
\hline
\end{tabular}

Para analizar las diferencias entre las carreras evaluadas, se implementó la prueba Tukey. Se encontró que los estudiantes de Ingeniería en Materiales obtuvieron promedios ponderados significativamente mayores que los estudiantes de IB y AE en las habilidades de razonamiento lógico $(p<.001)$, física ( $p<$ $.001)$ y química $(p<.001)$ y en las preferencias por tareas e intereses de Ingeniería en Materiales $(p<.001)$.

Por su parte, los estudiantes de Ingeniería en Biotecnología presentan promedios más altos que los estudiantes de AE e IM en la habilidad biológica $(p<.001)$ y en sus preferencias por los intereses $(p<$ $.001)$ y tareas $(p<.001)$ de Biotecnología. También, evidencian promedios mayores que los estudiantes de Administración de Empresas en las habilidades físicas $(p<.001)$ y químicas $(p<.001)$.

Finalmente, los estudiantes de Administración mostraron medias significativamente más altas que los estudiantes de IB e IM en la habilidad de liderazgo $(p<.001)$ y en las preferencias por tareas $(p<$ $.001)$ e intereses $(p<.001)$ de Administración. Debido a las diferencias evidenciadas por carreras, los análisis de varianza, según satisfacción vocacional, se realizaron por separado para cada carrera.

En el caso de Ingeniería en Materiales, los estudiantes más satisfechos obtienen promedios más altos en las habilidades químicas $\left(\mathrm{F}(2)=3.730, p=.028, \eta_{\mathrm{p}}{ }^{2}=.84\right)$, físicas $\left(\mathrm{F}(2)=3.376, p=.039, \eta_{\mathrm{p}}{ }^{2}\right.$ $=.76)$, de liderazgo $\left(\mathrm{F}(2)=8.141, p<.001, \eta_{\mathrm{p}}{ }^{2}=.13\right)$ y disciplina $\left(\mathrm{F}(2)=10.660, p<.001, \eta_{\mathrm{p}}{ }^{2}=.21\right)$.

En Ingeniería en Biotecnología se encontró que los estudiantes con mayores niveles de satisfacción vocacional obtienen promedios más altos en las habilidades biológicas $\left(\mathrm{F}(2)=6.020, p=.004, \eta_{\mathrm{p}}{ }^{2}=\right.$ 


\section{A. Alfaro-Barquero y S. Chinchilla-Brenes}

.12), liderazgo $\left(\mathrm{F}(2)=5.286, p=.007, \eta_{\mathrm{p}}{ }^{2}=.11\right)$ y disciplina $\left(\mathrm{F}(2)=9.967, \mathrm{p}=.001, \eta_{\mathrm{p}}{ }^{2}=.18\right)$, así como en intereses $\left(\mathrm{F}(2)=3.599, p=.03, \eta_{\mathrm{p}}{ }^{2}=.08\right)$ y tareas de $\mathrm{IB}\left(\mathrm{F}(2)=3.304, p=.041, \eta_{\mathrm{p}}{ }^{2}=.08\right)$.

En el caso de Administración de Empresas se evidenciaron diferencias significativas según el nivel de satisfacción vocacional en las habilidades de disciplina $\left(\mathrm{F}(2)=13.039, p=.001, \eta_{\mathrm{p}}{ }^{2}=.21\right)$, liderazgo $\left(\mathrm{F}(2)=5.203, p=.007, \eta_{\mathrm{p}}{ }^{2}=.12\right)$, tareas $\left(\mathrm{F}(2)=4.370, p=.015, \eta_{\mathrm{p}}{ }^{2}=.11\right)$ e intereses $(\mathrm{F}(2)=4.359, p=$ $\left..016, \eta_{\mathrm{p}}^{2}=.08\right)$ de Administración.

En relación con el sexo, se encontró que las mujeres obtienen promedios significativamente mayores en las habilidades biológicas $\left(t(280)=-2.688, p=.008, \eta_{\mathrm{p}}{ }^{2}=.05\right)$, en disciplina $(t(281)=-3.420$, $\left.p<.001, \eta_{\mathrm{p}}{ }^{2}=.05\right) \mathrm{y}$ en los intereses $\left(t(281)=-3.442, p<.001, \eta_{\mathrm{p}}{ }^{2}=.07\right)$, y tareas $(t(281)=-3.042, p=$ $\left..003, \eta_{\mathrm{p}}{ }^{2}=.05\right)$ de IB, así como en el promedio ponderado $\left(t(238)=-4.311, p<.001, \eta_{\mathrm{p}}{ }^{2}=.08\right)$ y en los niveles de satisfacción vocacional $\left(t(279)=-2.217, p<.001, \eta_{\mathrm{p}}^{2}=.03\right)$.

Por su parte, los hombres alcanzaron mayores promedios en las habilidades lógico-matemáticas $\left(t(281)=3.816, p<.001, \eta_{\mathrm{p}}{ }^{2}=.05\right)$ y física $\left(t(281)=3.948, p<.001, \eta_{\mathrm{p}}{ }^{2}=.08\right)$, así como en los intereses $\left(t(281)=3.534, p<.001, \eta_{\mathrm{p}}{ }^{2}=.08\right)$ y tareas $\left(t(281)=4.046, p<.001, \eta_{\mathrm{p}}{ }^{2}=.09\right)$ del área de IM.

No se evidenciaron diferencias según sexo en la habilidad química $\left(t(281)=1.749, p=.056, \eta_{\mathrm{p}}{ }^{2}=\right.$ $.01)$, en liderazgo $\left(t(281)=-.297, p=.766, \eta_{\mathrm{p}}{ }^{2}=.00\right)$, ni en los intereses $\left(t(281)=-.763, p=.446, \eta_{\mathrm{p}}{ }^{2}=\right.$ $.00)$ y tareas de $\operatorname{AE}\left(t(281)=-.373, p=.710, \eta_{\mathrm{p}}^{2}=.00\right)$.

\section{Discusión}

Desde el punto de vista psicométrico, la prueba vocacional mostró adecuados indicadores estadísticos y puede considerarse una herramienta útil para discriminar entre las preferencias y las habilidades vocacionales de los estudiantes de las tres carreras evaluadas. Presenta mejores evidencias de confiabilidad y validez que las escalas de habilidades y actividades del SDS de Holland, debido a su mayor grado de especificidad.

Los resultados aportan evidencias a favor del supuesto de la existencia de las diferencias entre perfiles vocacionales, aún y cuando se ubiquen dentro del área de ingeniería, tal es el caso de las carreras de Materiales y Biotecnología. Si bien estas evidencias son importantes, también resultan aún incipientes y frágiles. Señalan un amplio camino de investigación por venir y generan más interrogantes que respuestas.

Asimismo, se encontró que, en la mayoría de los casos, los estudiantes que muestran altos niveles de satisfacción tienden a obtener puntuaciones más altas en las habilidades, los intereses y las tareas requeridas en la carrera, lo que evidencia que la satisfacción vocacional refleja el grado de congruencia entre los intereses y las características de la carrera (Benítez, 2010).

En concordancia con los hallazgos de estudios que señalan la existencia de diferencias en las preferencias según sexo, en esta investigación se encontró que los hombres muestran preferencia por la Ingeniería en Materiales y se perciben con mayores habilidades en el área física y lógico-matemática, mientras que las mujeres lo hacen en la habilidad biológica y el campo de la Biotecnología, la disciplina, la satisfacción vocacional y el rendimiento académico (Alfaro-Barquero \& Chinchilla-Brenes, 2016 y 2017; Fogliatto, Pérez, Olaz \& Parodi, 2003; Martínez \& Ángeles 2016; Martínez-Martínez et al., 2016; Montero, 2005). 


\section{DISEÑO DE UN INSTRUMENTO DE PREFERENCIAS VOCACIONALES}

A pesar de que los hombres se autoevaluaron con mayores habilidades en las áreas de razonamiento lógico-matemático y física, las mujeres obtuvieron promedios ponderados más altos que los hombres, lo que refleja que las percepciones sexistas siguen muy impregnadas en la población y las mujeres se conciben con menores habilidades para el área de ingeniería. Así, tal y como lo señala Cepero (2009), son los intereses, las preferencias, en conjunto con el sexo, las variables de gran relevancia en la elección vocacional.

Es importante destacar que no se evidenciaron diferencias por sexo en las preferencias por el área de Administración. Lo anterior coincide con los resultados reportados por Parreaguirre (2016) al ser el área de las finanzas donde existe mayor paridad en el mercado laboral en Costa Rica.

Como limitación cabe señalar que no fue posible obtener un mayor número de muestra en virtud del tamaño de la población de Biotecnología y Materiales.

\section{Referencias}

Adelfang, J. (8 de marzo, 2016). Microanálisis macro. Los datos de hoy en menos de 200 palabras. La República, p. 20.

Alfaro-Barquero, A., \& Chinchilla-Brenes, S. (2017). Construcción y Validación de un instrumento de evaluación de preferencias y habilidades vocacionales para carreras científico-tecnológicas. Tecnología en Marcha, 30(4), 139-149.

Alfaro-Barquero A., \& Chinchilla-Brenes, S. (2016). Reflexiones en torno a la condición de género en los perfiles vocacionales de algunas carreras de ingeniería en el Instituto Tecnológico de Costa Rica (ITCR). Trabajo presentado en XI Congreso Iberoamericano de Ciencia, Tecnología y Género. San José, Costa Rica.

Álvarez, J., Aguilar, J. M., Fernández, J. M., \& Sicilia, M. (2014). El prestigio profesional y social: determinante de la decisión vocacional. Revista Española de Orientación y Psicopedagogía, 25(2), 44-50.

Arcega, R. (2016). Habilidades del pensamiento y su relación con Matemáticas. Revista Iberoamericana de Producción Académica y Gestión Educativa, (2), 1-24.

Barrera, M. (2016). Orientación vocacional para una acertada elección de carreras en la educación superior. Mucutíes Universitaria, 3, 25-34.

Benítez, J. (2010). Madurez Vocacional y satisfacción académica de los estudiantes de enfermería de Luz (tesis de maestría). Recuperada de http://tesis.luz.edu.ve/tde arquivos/111/TDE-2011-05-31T11:34:11Z-1029/ Publico/Benitez_Chirino_Juana_Gregoria.pdf

Bohoslavsky, R. (1984). Orientación Vocacional: la estrategia clínica. Buenos Aires: Ediciones Nueva Visión.

Carrasco, E., Zúñiga, C., \& Espinoza, J. (2014). Elección de carrera en estudiantes de nivel socioeconómico bajo de universidades chilenas altamente selectivas. Calidad en la Educación, 40, 95-128.

Cea, M. (2002). Análisis Multivariante. Teoría y práctica en la investigación social. Editorial Síntesis. Madrid.

Cepero, A. (2009). Las preferencias profesionales y vocacionales del alumnado de secundaria y formación profesional especifica (tesis doctoral). Universidad de Granada. España. 


\section{A. Alfaro-Barquero y S. Chinchilla-Brenes}

Díaz-Barajas, D., Morales-Rodríguez, M., \& Amador- Zavala, L. (2009). Perfil vocacional y rendimiento escolar en universitarios. Revista Mexicana de Orientación Educativa, 6(16), 20-23.

Donoso-Vázquez, T. (octubre, 2012). Propuestas para una orientación con perspectiva de género. Ponencia presentada en el simposio del I Congreso internacional e interuniversitario de Orientación Educativa y Profesional. Rol y retos de la orientación en la universidad y en la sociedad del siglo XXI, Málaga.

Echavarri, M., Godoy, J., \& Olaz, F. (2007). Diferencias de género en habilidades cognitivas y rendimiento académico en estudiantes universitarios. Univ. Psychol. Bogotá (Colombia), 6(2), 319-329.

Espíndola, M. (2015). Caracterización de factores: psicológico, social, económico y académico, asociados a la elección vocacional en estudiantes inscritos al curso de orientación vocacional de la UAJMS1. Revista Ventana Cientifica, 6(23), 23-30.

Estrada, P. (2011). Factores que intervienen en la elección de carrera de estudiantes de bachillerado de dos modalidades educativas. XI Congreso Nacional de Investigación Educativa. México.

Fogliatto, H., Pérez, E., Olaz, F., \& Parodi, L. (2003). Cuestionario de Intereses Profesionales Revisado (CIP-R). Análisis de Propiedades Psicométricas. Evaluar, 3, 61-79.

Hansen, J. (2004). Interest Inventories.In G. Goldstein \& M. Hersen (Eds.). (3a.ed.), Handbook of psychological assessment (13-3). New York: Academic Press.

Hernández, V. (2001). Análisis causal de los intereses profesionales en los estudiante de secundaria (tesis doctoral). Universidad Cumplutense de Madrid, España. Recuperada de https://eprints.ucm.es/4860/

Herrera, E., \& Burgoa, T. (2012). La importancia del perfil vocacional en la visión del estudiante universitario: caso de estudio en facultades de la Universidad Autónoma de Nueva León, UANL. DAENA: International Journal of Good Conscience, 8(2), 23-47.

Holland, J. (1994). Self-directed search. Forma R. (4a.ed.). Psychological Assesment Resources. Florida.

Martelo, R., Jiménez-Pitre, I., \& Quintana, A. (2018). Determinación del Perfil Profesional de Estudiantes de Pregrado Aplicando la Técnica de Análisis Comparativo. Información tecnológica, 29(2), 29-40.

Martínez, J., \& Ángeles, I. (2016). Características de los intereses vocacionales en alumnos de educación secundaria evaluados a través del explora. International Journal of Developmental and Educational Psychology, 3(1), $549-562$.

Martínez. J. (2016). Explora cuestionario para la orientación vocacional y profesional. International Journal of Developmental and Educational Psychology, 4(1), 335-344.

Martínez-Martínez, A., Zurita-Ortega, F., Castro-Sánchez, M., Chacón-Cuberos, R., Hinojo-Lucena, M., \& Espejo-Garcés, T. (2016). La elección de estudio superiores universitarios en estudiantes de último curso de bachillerato y ciclos formativos. Revista Electrónica Educare, 20(1), 304-321.

Montero, P. (2005). Actualización del Inventario de Intereses Vocacionales de G. F. Kuder forma C, en estudiantes de II año de enseñanza media Científico-Humanista del Gran Santiago (tesis de licenciatura). Universidad de Chile, Santiago. 


\section{DISEÑO DE UN INSTRUMENTO DE PREFERENCIAS VOCACIONALES}

Müller, M. (1992). Orientación vocacional. Buenos Aires: Miño y Dávila Editores.

Pacheco, E., \& Blanco, M. (2015). Metodología mixta: su aplicación en México en el campo de la demografía. Estudios demográficos y urbanos, 30(3), 725-770.

Parreaguirre, F. (8 de marzo, 2016). Empoderamiento femenino Bancario es la tendencia. La República, p. 18-19.

Pérez, E., Cupani, M. \& Ayllón, S. (2005). Predictores de rendimiento académico en la escuela media: habilidades, autoeficacia y rasgos de personalidad. Avaliação Psicológica, 4(1), 1-11.

Restrepo, B., Carvajal C., \& Roldán, N. (2016). Preferencias profesionales de estudiantes de educación media del Bajo Cauca, Norte y Nordeste de Antioquia, Colombia. Senderos pedagógicos, 7, 95-105.

Rodríguez, M. (2002). Hacia una nueva orientación universitaria: modelos integrados de acción tutoría, orientación curricular y construcción del proyecto profesional. Barcelona: Ediciones de la Universitat de Barcelona.

Rodríguez, R. (8 de marzo, 2016). Gerencia femenina, fórmula clave para éxito empresarial. La República, p. 8.

Rosado, C., \& María, A. (2012). Género, orientación educativa y profesional. Revista Mexicana de Orientación Educativa, 9(22), 36-41.

Sevilla, D., Puerta, V., \& Dávila, J. (2010). Influencia de los factores socioeconómicos en la deserción estudiantil de la carrera de Ciencias Sociales. Ciencia e interculturalidad, 6(1), 72-84.

Urribarrí, L, Flores, Y., \& Añez, I. (2015). Un proceso grupal para la atención vocacional: Éxito, Satisfacción y Estabilidad Vocacional de los estudiantes universitarios. Encuentro Educacional, 20(2), 319-336.

Vargas, H., \& Huamán, I. (2011). Características de los intereses vocacionales de los alumnos del 2do año y 3er año de la escuela de ingeniería en informática y sistemas de la facultad de ciencias de la Universidad Nacional Jorge Basadre Grohmann de Tacna-año académico 2009. Ciencia y Desarrollo, 13, 68-71.

Woyno, W., \& Oñoro, R. (2005). Escala de preferencias Kuder Vocacional. Manual Moderno. México.

Recibido: 22 de Marzo de 2018

Revisión recibida: 10 de Octubre de 2019

Aceptado: 11 de Noviembre de 2019 


\section{Sobre las autoras:}

Alejandra Alfaro-Barquero es máster en Psicología, docente y asesora psicoeducativa en el Instituto Tecnológico de Costa Rica (ITCR) desde el 2002. Forma parte del Programa de Investigación Educativa en el Departamento de Orientación y Psicología. Es miembro del Comité Científico de la Revista digital "Matemática, Educación e Internet" desde el 2015 y de la Revista de Comunicación a partir del 2017. Cuenta con cerca de 15 publicaciones y conferencias en congresos nacionales e internacionales.

Sonia Chinchilla-Brenes labora como psicóloga. Se desempeña como funcionaria del Departamento de Orientación y Psicología del Instituto Tecnológico de Costa Rica desde 1990. Es integrante del Comité Técnico de Investigación del Departamento. Ha participado en proyectos de investigación educativa y en diversos programas de apoyo estudiantil. Ha elaborado numeroso material para el estudiantado y ha participado en, al menos, veinte eventos de carácter nacional e internacional como ponente tanto de sus experiencias de trabajo como de los resultados de las investigaciones en las que ha participado. 